\title{
Novel three-dimensional renewable phytic acid based aerogel electronics
}

\author{
Rui Yao*
}

China Electronics Technology Group Corp 38th Research Institute, Hefei 230088, China

\begin{abstract}
Novel three-dimensional renewable phytic acid (AP) aerogel electronics were synthesized through in-site polymerization and oriented lyophilization method. Three stages of the aerogels were apparently observed via SEM and TEM tests: the honeycomb structure consituted the first stage; the network with micropores formed the second stage; the third stage was composed of nanopores structure inside the micropores. When the molar ratio of aniline and phytic acid reached certain degree, the AP aerogel will emerge high pore volume and excellent specific capacitance. What's more, the mechanical properties of AP aerogel were about 5 times ( 9 times for electrical properties) higher in vertical direction than those in horizontal direction. To sum up, the three-dimensional AP aerogel owned great mechanical and electrical properties in specific direction, at the same time, it exhibited outstanding specific capacitance (448 F. $\left.\mathrm{g}^{-1}\right)$.
\end{abstract}

Keywords: Renewable phytic acid; Three-dimensional; Aerogel electronics; Mechanical performance; Electrical performance.

\section{Introduction}

Phytic acid which can be extracted from plants is environmentally friendly and renewable ${ }^{1}$, while it does not exhibit good electrical performance. On the contrary, polyaniline (PANI) with certain virulence owns electrical conductivity ${ }^{2}$. In addition, PANI based aerogels present more excellent electrical capacity ${ }^{3}$. When combining phytic acid with PANI to form aerogel, the virulence of PANI will disappear and the aerogel gains much better electrical properties than single PANI ${ }^{4,5}$. The traditional method to prepare the aniline/phytic acid(AP) aerogels is freeze-drying, however, the aerogels made by this method display mediocre electrical performance together with low compressive strength ${ }^{6,7}$. In this study, a oriented lyophilization method were used to prepare the AP aerogels, which made them formed honeycomb structure. This structure resulted in much higher electrical properties of the aerogels in specific direction, simultaneously, given rise to higher compressive strength ${ }^{8}$. In addition, the aerogels also exhibit outstanding specific capacitance. So, in the fields of electrical components, such as battery and capacitor, they will certainly have great application prospect ${ }^{9-11}$.

\footnotetext{
*Corresponding author:277462297@qq.com
} 


\section{Experimental}

Figure 1 illustrated the three step method of preparing AP aerogel. The steps were detailedly shown: First, stirring $0.5 \mathrm{mmol}$ phytic acid into $2 \mathrm{~mL}$ of deionized (DI) water, and solution A was gained when it's adequately dissolved. Then, adding $2.5 \mathrm{mmol}$ aniline monomer (distilled prior) to solution A which will form a clear solution after ultrasonic processing. At last, solution B was obtained after $0.625 \mathrm{mmol}$ ammonium persulfate was dissolved in DI water $(0.5 \mathrm{~mL})$. Second, mixing Solution $\mathrm{A}$ and $\mathrm{B}$ into the polydimethylsiloxane(PDMS) mold quickly (the hydrogel was formed within $3 \mathrm{~min}$, and its color turned into dark green), then immersing the bottom of mold into liquid nitrogen for refrigeration. Third, the frozen hydrogels were lyophilized at $-50^{\circ} \mathrm{C}$ for $24 \mathrm{~h}^{12,13}$, and AP aerogel samples (AP1) were obtained after the redundant ion were wiped off by washing and high-temperature $\left(60^{\circ} \mathrm{C}\right)$ drying. In addition, in order to comparing with AP1 aerogels, normal lyophilized AP1 (N-AP1), AP2 (0.25 mmol phytic acid; $2.75 \mathrm{mmol}$ aniline) and AP3 (1 mmol phytic acid; $2 \mathrm{mmol}$ aniline) were prepared.

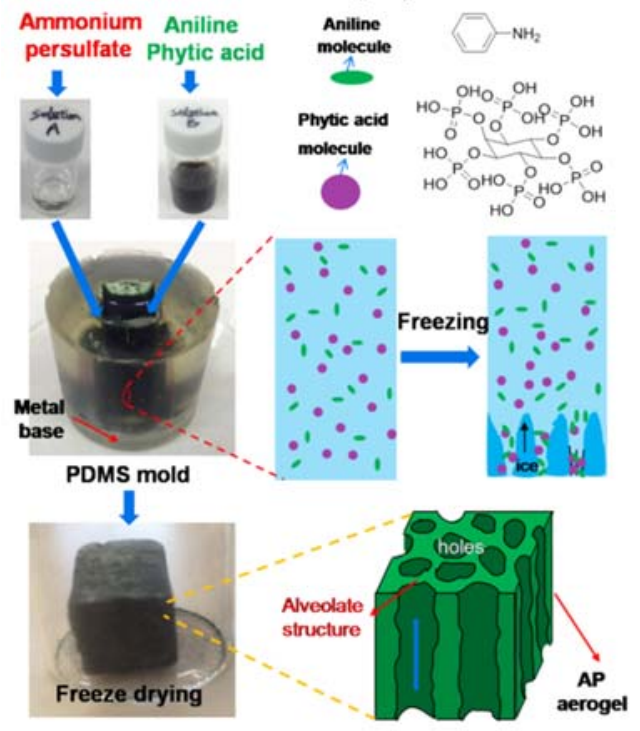

Fig. 1. The preparation method and structural representation of AP aerogel.

\section{Results and discussion}

The three step method to synthesize the aerogels is distinctly displayed in experimental section. The PDMS mold has protected the broadside of the hydrogel from been frozen when immersing its bottom into liquid nitrogen, the hydrogel heat will not delivery from the surround to outside, while the metal base with good specific heat capacity transmits the heat quickly, hence, ice crystal will germinate along the spread path from the bottom as shown in Figure 1. Under oriented function of ice crystal the molecules will grow into alveolate structure with AP network(Figure 2(c)). Finally, the three-dimensional AP aerogel is acquired through the freeze-drying method. In addition, the -OH broad peak caused by phytic acid can be observed from the FTIR curve of AP aerogel(Figure 2(e)). Specifically, the three-dimensional structure is composed of three stages: the honeycomb structure consituted the first stage; the network with micropores formed the second stage; the third stage was composed of nanopores structure inside the micropores. 

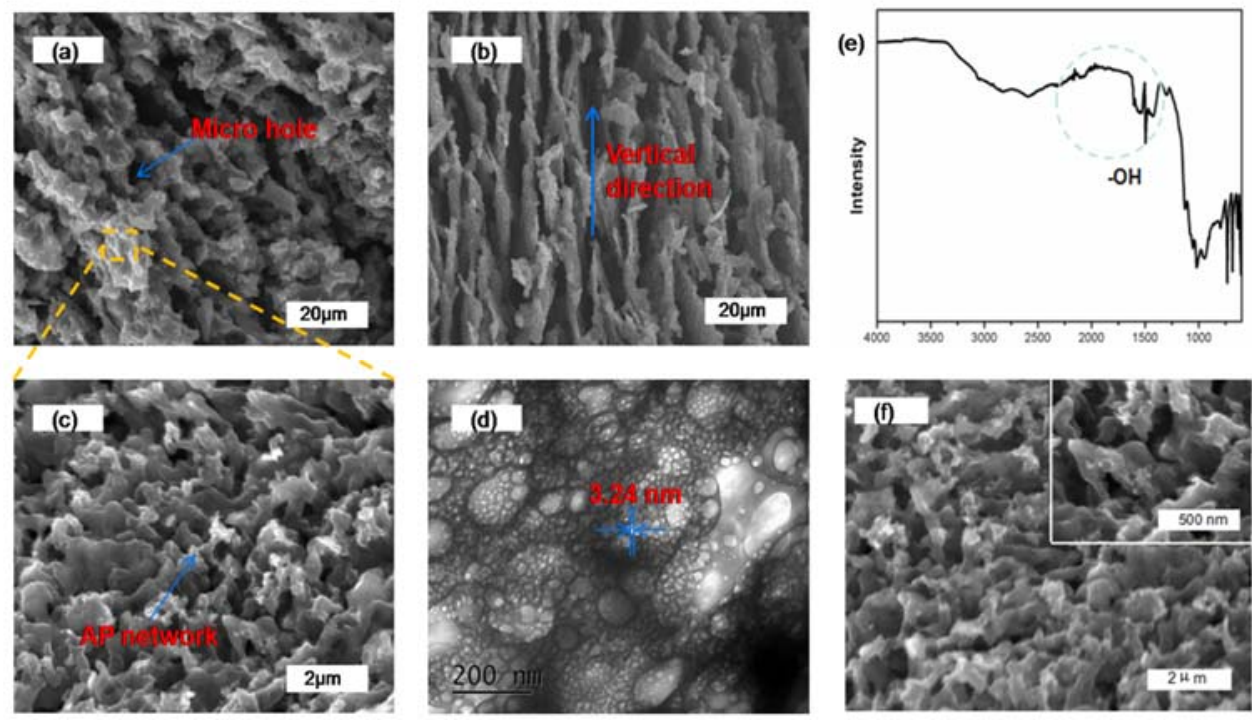

Fig. 2. The SEM, FTIR and TEM images of AP1 aerogels: (a) top SEM image; (b) side SEM image; (c) SEM image of Micropores; (d) TEM image of Nanopores; (e)FTIR curve; (f) The SEM images of N-AP1 aerogels.

The top SEM image of AP1 aerogel is displayed in Figure 2(a), while its side SEM image is shown in Figure 2(b). Combining the image of top and side together, the honeycomb structure is observed. In addition, compared with structure of AP2 and AP3(Figure 3), AP1 aerogel expresses more balance and stable: the AP1 has much uniform structure, while there exist huge holes (the blue circles) on the top SEM images of AP2 and AP3 which are confirmed to decline the stability of the aerogels. Ulteriorly blowing up the top image of AP1 in Figure 2(c)and(f), Micro holes AP network(second-stage) is evidently surveyed. Further observing the structure through TEM in Figure 2 (d), the $3 \mathrm{~nm}$ diameter nano-pores of AP aerogel are clearly demonstrated.
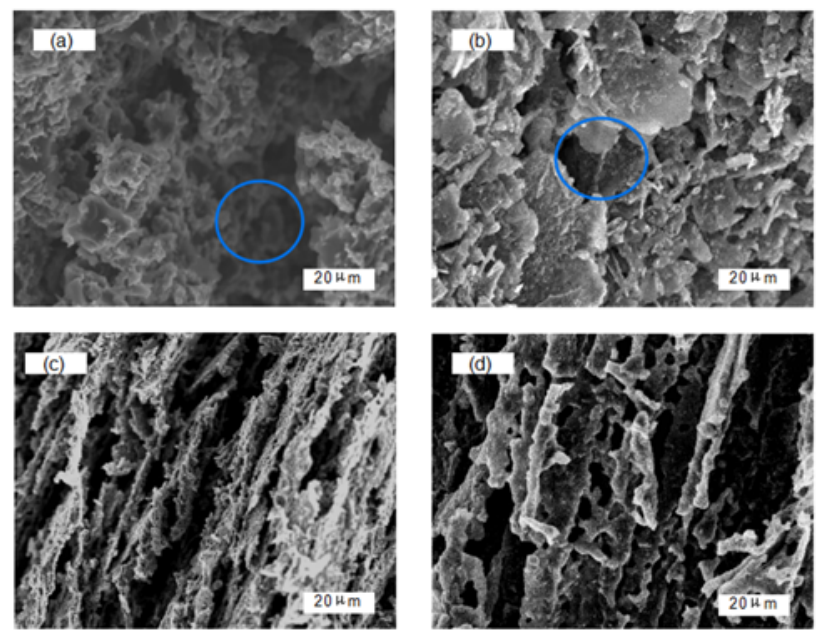

Fig. 3. The SEM images: (a) AP2 top; (b) AP3 top; (c) AP2 side; (d) AP3 side. 
In Figure 4 (a), AP aerogels exhibit broad peaks which means that the aerogels are organic materials. Seen from the XRD curves, the imaginary line enclose the small angle peaks $\left(2 \theta=5.7^{\circ}\right)$ which expresses that the aerogels contain nanopores. Calculating through the Bragg equation $(2 \mathrm{~d} \sin \theta=\mathrm{n} \lambda)$, the width of the nano-pores is about $3.02 \mathrm{~nm}$. In addition, viewed from the BET curves in Figure 4 (b), the aerogels highest pore volume are concentrated in $2.89 \mathrm{~nm}$, and this result is much accordant with the TEM image. What's more, it's evident that AP1 own the highest pore volume compared with AP1 and AP2 through the intensity of the peaks. At last, characteristic peaks $(291 \mathrm{~nm}$ and $289 \mathrm{~nm}$ in ultraviolet region) can be detected from AP aerogel and phytic acid by UV/Vis-NIR (Figure 4(c)). AP aerogels are proved to be gained successfully through above analysis.
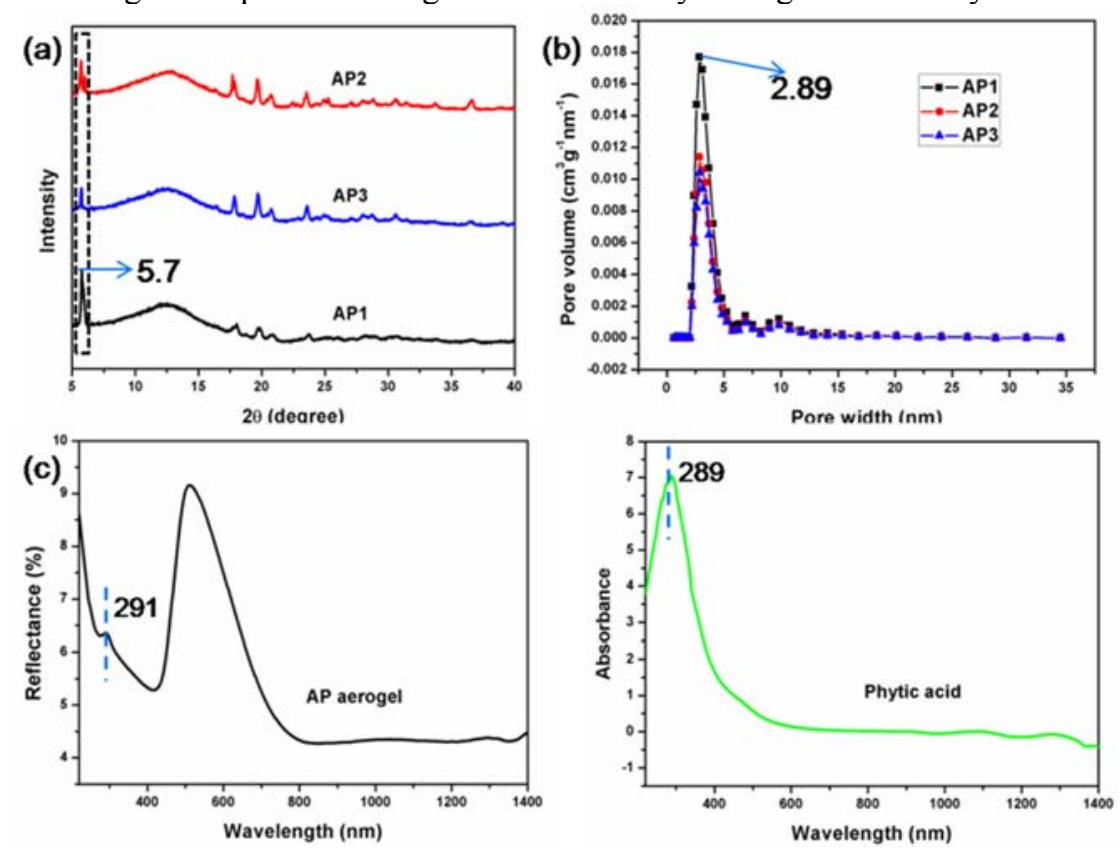

Fig. 4. The XRD, BET and UV/Vis-NIR curves of AP aerogels and phytic acid (a) XRD curves (b) BET curves (c)The UV/Vis-NIR of AP aerogel(Solid-state) and Phytic acid(Liquid-state).

AP aerogels were made into active supercapacitor electrodes to assess the electrical performance, a galvanostatic discharge test was implemented in a traditional three-electrode system (Observed from Figure 5(c)). Figure 5(a) exhibits the specific capacitance results of the aerogels, among them, the value of AP1 is $448{\mathrm{~F} . \mathrm{g}^{-1}}^{-1}(0.5 \mathrm{~A} / \mathrm{g}$ current density) which is much higher than that of AP2 and AP3, and it's attributed to the nano-pores structure(third stage) ${ }^{14}$. To be specific, AP1 with great pore volume forms much larger specific surface area which will certainly enhance the energy capacity by more storing space.

Viewed from Figure 5 (b), the mechanical properties of AP aerogel were about 5 times (9 times for electrical properties) higher in vertical direction(Figure 2(b)) than those in horizontal direction. What's more, compared with N-AP(isotropous in all directions), the AP1 aerogel also exhibits much higher mechanical and electrical performances in vertical direction. The mechanism for higher performance in certain direction is analysed as follows: First, the three-dimensional honeycomb structure owns stronger bearing power in vertical direction caused by the oriented function, which will surely increase its mechanical performance. Second, the AP network contains doped ions which offers the electric conductivity of AP aerogel. In vertical direction, a great many tiny parallel circults of doped ions constitute big conductive network, which will reduce the resistance observably. 

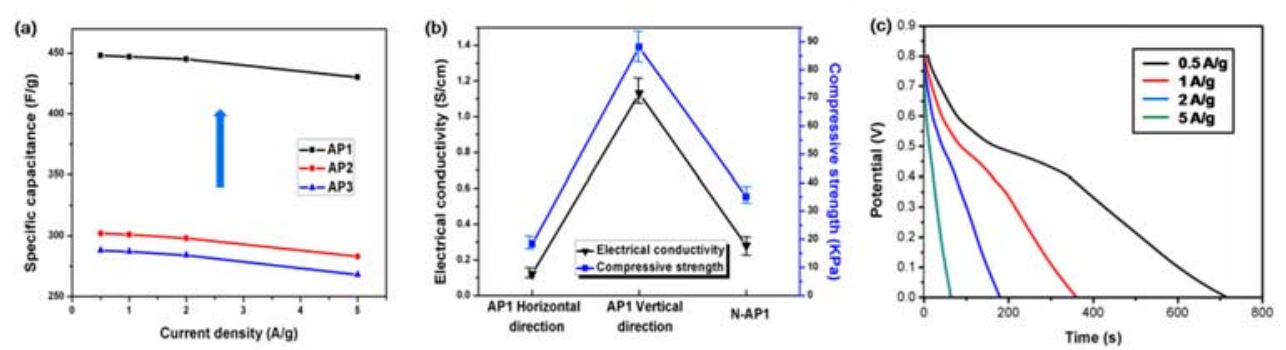

Fig. 5. The mechanical and electrical performance of AP aerogels: (a) Specific capacitance; (b) Compressive strength and electrical conductivities; (c)Galvanostatic discharge profiles.

\section{Conclusions}

In this paper, novel three-dimensional renewable phytic acid based aerogel electronics with micro and nano pores were prepared with good comprehensive performance: For mechanical performance, the compressive strength of AP aerogel exhibited about 5 times higher in vertical direction than those in horizontal direction. For electrical performance, the increasing rate of electrical conductivities was 9 times. To sum up, the threedimensional AP aerogel owned great mechanical and electrical properties in specific direction, at the same time, it exhibited outstanding specific capacitance $\left(448 \mathrm{~F}^{-1} \mathrm{~g}^{-1}\right)$.

\section{References}

1. E. Feizollahi, R.S. Mirmahdi, A. Zoghi, R.T. Zijlstra, M.S.Roopesh, T. Vasanthan, Food Res. Int, 143, 110284(2021).

2. B. Song, C.C. Tuan, X.G. Huang, L.Y. Li, K.S. Moon, C.P. Wong, Mater lett., 166, 12 (2016).

3. Y.N. Meng, K. Wang, Y.J. Zhang, Z.X. Wei, Adv Mater, 25, 6985 (2013).

4. G.P. Krishnaa, P. Navaneeth, T. Ramachandran, Mater Today, 33, 2407 (2020).

5. H.X. Yang, N. Wang, Y.M. Ren, L. Cai, Z.M. Chen, Q. Xu, Mater lett., 139, 471 (2015)-474.

6. K.A. Milakin, Z. Morávková, U. Acharya, Polymer, 217, 123450(2021).

7. Y.S. Hao, Y.F. Zhao, X.X. Yang, Corros Sci, 151, 175(2019).

8. W.J. Jiang, W.J. Luo, R.L. Zong, W.Q. Yao, Z.P. Li, Y.F. Zhu, Small, 12, 4370(2016).

9. W.W. Li, F.X. Gao, X.Q. Wang, N. Zhang, M.M. Ma, Angew Chem Int Ed, 55, 9196 (2016).

10. D.Y. Zhai, B.R. Liu, Y. Shi, L.J. Pan, W.B. Li, et al, ACS Nano, 7, 3540 (2013).

11. K. Mohan, A. Bora, R.S. Roy, B.C. Nath, S.K. Dolui, Sol Energy. 186, 360 (2019) .

12. F. He, C. Sui, X.D. He, M.W. Li, Mater Lett., 152, 9 (2015).

13. L.J. Pan, G.H. Yu, D.Y. Zhai, et al, PNAS, 109, 9287 (2012).

14. G.Q. Tang, Z.G. Jiang, X.F. Li, H.B. Zhang, D. Aravind, Carbon, 77, 592 (2014). 